\title{
Correlated Topological States in Graphene Nanoribbon Heterostructures
}

\author{
Joost, Jan-Philip; Jauho, Antti-Pekka; Bonitz, Michael
}

Published in:

Nano Letters

Link to article, DOI:

10.1021/acs.nanolett.9b04075

Publication date:

2019

Document Version

Publisher's PDF, also known as Version of record

Link back to DTU Orbit

Citation (APA):

Joost, J-P., Jauho, A-P., \& Bonitz, M. (2019). Correlated Topological States in Graphene Nanoribbon Heterostructures. Nano Letters, 19(12), 9045-9050. https://doi.org/10.1021/acs.nanolett.9b04075

\section{General rights}

Copyright and moral rights for the publications made accessible in the public portal are retained by the authors and/or other copyright owners and it is a condition of accessing publications that users recognise and abide by the legal requirements associated with these rights.

- Users may download and print one copy of any publication from the public portal for the purpose of private study or research.

- You may not further distribute the material or use it for any profit-making activity or commercial gain

- You may freely distribute the URL identifying the publication in the public portal

If you believe that this document breaches copyright please contact us providing details, and we will remove access to the work immediately and investigate your claim. 


\title{
Correlated Topological States in Graphene Nanoribbon Heterostructures
}

\author{
Jan-Philip Joost, ${ }^{\dagger}$ Antti-Pekka Jauho, ${ }^{\dagger}$ and Michael Bonitz ${ }^{*}{ }^{\dagger} \odot$ \\ ${ }^{\dagger}$ Institut für Theoretische Physik und Astrophysik, Christian-Albrechts-Universität zu Kiel, D-24098 Kiel, Germany \\ ‡CNG, DTU Physics, Technical University of Denmark, Kongens Lyngby, DK 2800, Denmark
}

\section{Supporting Information}

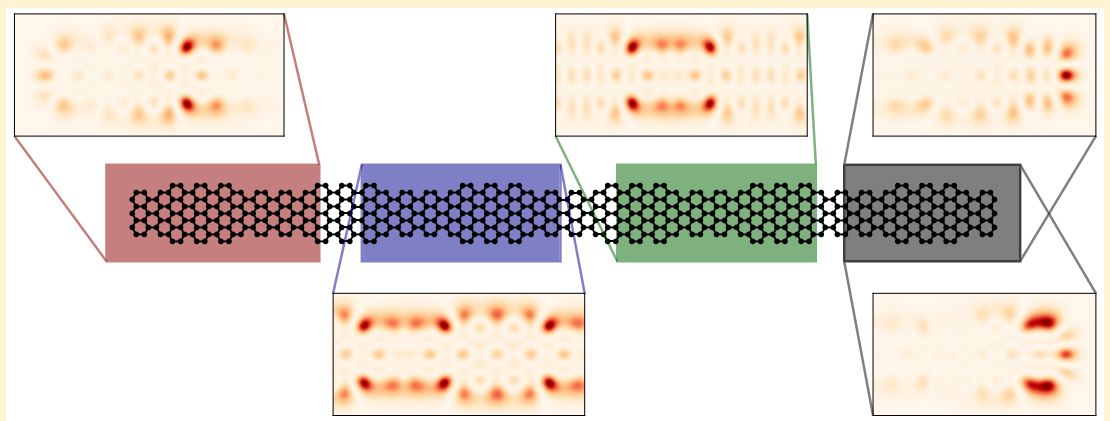

ABSTRACT: Finite graphene nanoribbon (GNR) heterostructures host intriguing topological in-gap states (Rizzo, D. J.; et al. Nature 2018, 560, 204). These states may be localized either at the bulk edges or at the ends of the structure. Here we show that correlation effects (not included in previous density functional simulations) play a key role in these systems: they result in increased magnetic moments at the ribbon edges accompanied by a significant energy renormalization of the topological end states, even in the presence of a metallic substrate. Our computed results are in excellent agreement with the experiments. Furthermore, we discover a striking, novel mechanism that causes an energy splitting of the nonzero-energy topological end states for a weakly screened system. We predict that similar effects should be observable in other GNR heterostructures as well.

KEYWORDS: Graphene nanoribbons, heterostructures, topological states, electronic correlations, Green function theory

trongly correlated materials host exciting physics such as $\checkmark$ superconductivity or the fractional quantum Hall effect. ${ }^{1}$ While in monolayer graphene electron correlations are weak, ${ }^{2}$ carbon based finite systems and heterostructures can exhibit flat bands near the Fermi energy resulting in nontrivial correlated phases., One example is magic-angle twisted bilayer graphene where localized electrons lead to Mott-like insulator states and unconventional superconductivity. ${ }^{5-7}$ Another exciting class of systems that has been predicted to host strongly localized phases includes graphene nanoribbon (GNR) heterostructures. ${ }^{8-14}$ Similar to topological insulators, they combine an insulating bulk with robust in-gap boundary states ${ }^{15,16}$ and are expected to host Majorana Fermions in close proximity to a superconductor. ${ }^{17}$ Recently, it was confirmed that GNR heterostructures composed of alternating segments of 7- and 9-armchair GNRs (AGNRs), as sketched in Figure 1a, exhibit new topological bulk bands and end states that differ qualitatively from the band structures of pristine 7 - and 9-AGNRs. ${ }^{18}$

Although electronic correlations are expected to play a crucial role for the localized topological states in GNR heterostructures, $^{19}$ so far, most theoretical work has been restricted to tight-binding (TB) models or density functional theory within the local density approximation (LDA-DFT), which are known to completely ignore or underestimate these effects. Here, we present a systematic analysis of electronic correlations in 7-9-AGNRs, based on a Green functions method with $G W$ self-energy ${ }^{20,21}$ applied to an effective Hubbard model. We compute the differential conductance and find excellent agreement with the experimental measurements of ref 18. Our calculations reveal that, even in the presence of a screening $\mathrm{Au}(111)$ surface, local electronic correlations induce a strong energy renormalization of the band structure, especially the topological end states localized at the heterostructure-vacuum boundary experience strong quasiparticle corrections that are not captured by LDA-DFT. For freestanding systems, or systems on an insulating surface, we predict that these states exhibit an energy splitting due to a magnetic instability at the Fermi energy. The local buildup of electronic correlations is further analyzed by considering the local magnetic moment at the ribbon edges. We also examine finite size effects and the origin of the topological end states by varying the system size and end configuration, respectively. On this basis, we predict that a whole class of systems exists that can host end states with similar exciting properties.

Received: October 3, 2019

Revised: November 13, 2019

Published: November 18, 2019 

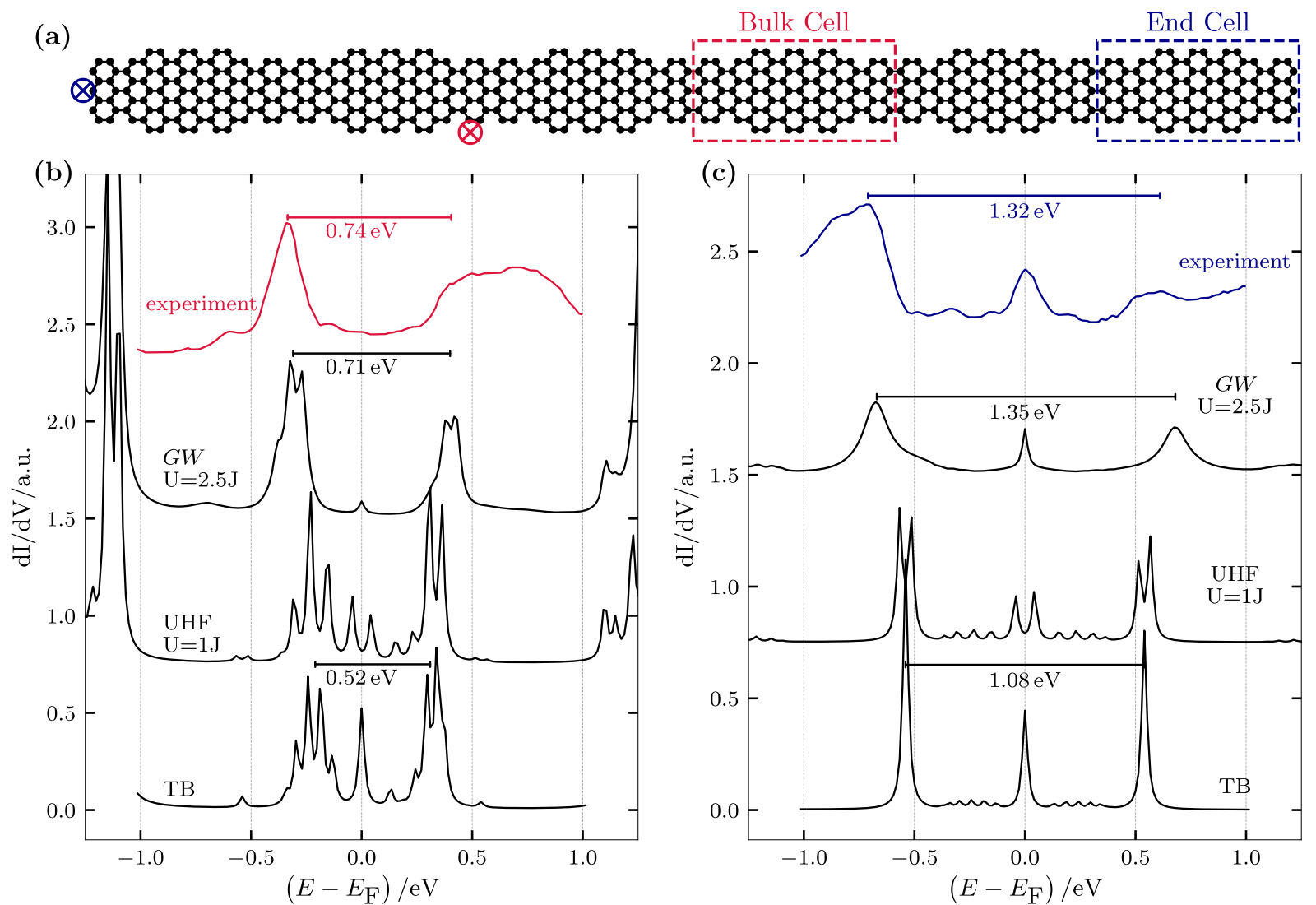

Figure 1. (a) 7-9-AGNR heterostructure containing six unit cells. The red [blue] cross marks the position of the $\mathrm{d} I / \mathrm{d} V$ spectra shown in (b) $[(\mathrm{c})]$. The red (blue) dashed rectangle marks the bulk (end) unit cell referenced in Figure 3. (b) $\mathrm{d} I / \mathrm{d} V$ spectrum measured (red) and simulated (black) at the position in the bulk region marked by the red cross in (a). (c) $\mathrm{d} I / \mathrm{d} V$ spectrum measured (blue) and simulated (black) at the position in the end region marked by the blue cross in (a). The curves in (b) and (c) are shifted vertically for better comparison. The experimental data are taken from ref 18 and corrected for charge doping effects.

Model. We consider the 7-9-AGNR heterostructure consisting of alternating 7-AGNR and 9-AGNR segments as depicted in Figure 1a. The system was realized experimentally on a metallic $\mathrm{Au}(111)$ surface by Rizzo et al. ${ }^{18}$ who observed topological in-gap states at the heterojunction between 7 - and 9-AGNR segments (bulk), and at the termini of the heterostructure (end). While previous LDA-DFT simulations describe the bulk bands reasonably well, they do not reproduce quantitatively the experimental energies of the end states (see further). To overcome these limitations, we apply a recently developed Green functions approach that gives access to spectral and magnetic properties of the system, see, for example, refs 20,22, and 23. The electronic system is described with an effective Hubbard model, the Hamiltonian of which is expressed in terms of the operators $\hat{c}_{i \alpha}^{\dagger}$ and $\hat{c}_{j \alpha}$ that create and annihilate an electron with spin projection $\alpha$ at site $i$ and $j$, respectively,

$$
\hat{H}=-J \sum_{\langle i, j\rangle, \alpha} \hat{c}_{i \alpha}^{\dagger} \hat{c}_{j \alpha}+U \sum_{i} \hat{c}_{i \uparrow}^{\dagger} \hat{c}_{i \uparrow} \hat{c}_{i \downarrow}^{\dagger} \hat{c}_{i \downarrow}
$$

where $J=2.7 \mathrm{eV}$ is the hopping amplitude between adjacent lattice sites, ${ }^{24}$ and $U$ is the on-site interaction. The edges of the GNR are assumed to be H-passivated. Observables can be computed from the Green function $G(\omega)$ that is defined in terms of the operators $\hat{c}_{i \alpha}^{\dagger}$ and $\hat{c}_{j \alpha}$; for details, see the Supporting Information (SI). An approximate solution of eq 1 is obtained by solving the self-consistent Dyson equation: ${ }^{22,23}$

$$
\boldsymbol{G}(\omega)=\boldsymbol{G}_{0}(\omega)+\boldsymbol{G}_{0}(\omega) \boldsymbol{\Sigma}(\omega) \boldsymbol{G}(\omega)
$$

where the single-particle Green function $\boldsymbol{G}$ contains the spectral and magnetic information on the system, and $G_{0}$ is its noninteracting limit. Correlation effects are included via the self-energy $\boldsymbol{\Sigma}$. In the present work, we report the first full $G W$ simulations of the system (eqs 1 and 2) for experimentally realized GNR heterostructures, as the one shown in Figure 1a. The details of the numerical procedure are provided in the SI.

In what follows, we compare the tight-binding (TB), unrestricted Hartree-Fock (UHF), and fully self-consistent $G W$ approximations for $\boldsymbol{\Sigma}$ to quantify electronic correlation effects. The TB approach, corresponding to setting $U=0$, is often used to describe GNRs, due to its simplicity, ${ }^{17,25-27}$ and here serves as a point of reference for the uncorrelated system. For $G W$, the on-site interaction was chosen such that it reproduces the experimental bulk band gap of ref 18 , resulting in $U=2.5 \mathrm{~J}$; see SI. This choice of $U$ also takes into account screening effects of the metallic substrate. The description of free-standing GNRs within GW requires a larger on-site interaction, ${ }^{28}$ which makes the self-consistent solution of eq 2 more challenging. Nonetheless, to get a qualitative understanding of the properties of free-standing heterojunctions, we employ the UHF approximation, which is known to qualitatively describe edge magnetism in free-standing ZGNRs. For this case, the on-site interaction was chosen as $U=1 J .{ }^{29}$ The spatially resolved $\mathrm{d} I / \mathrm{d} V$ data, recorded in an STM experiment, are generated by placing $2 \mathrm{p}_{z}$ orbitals on top 
(a)
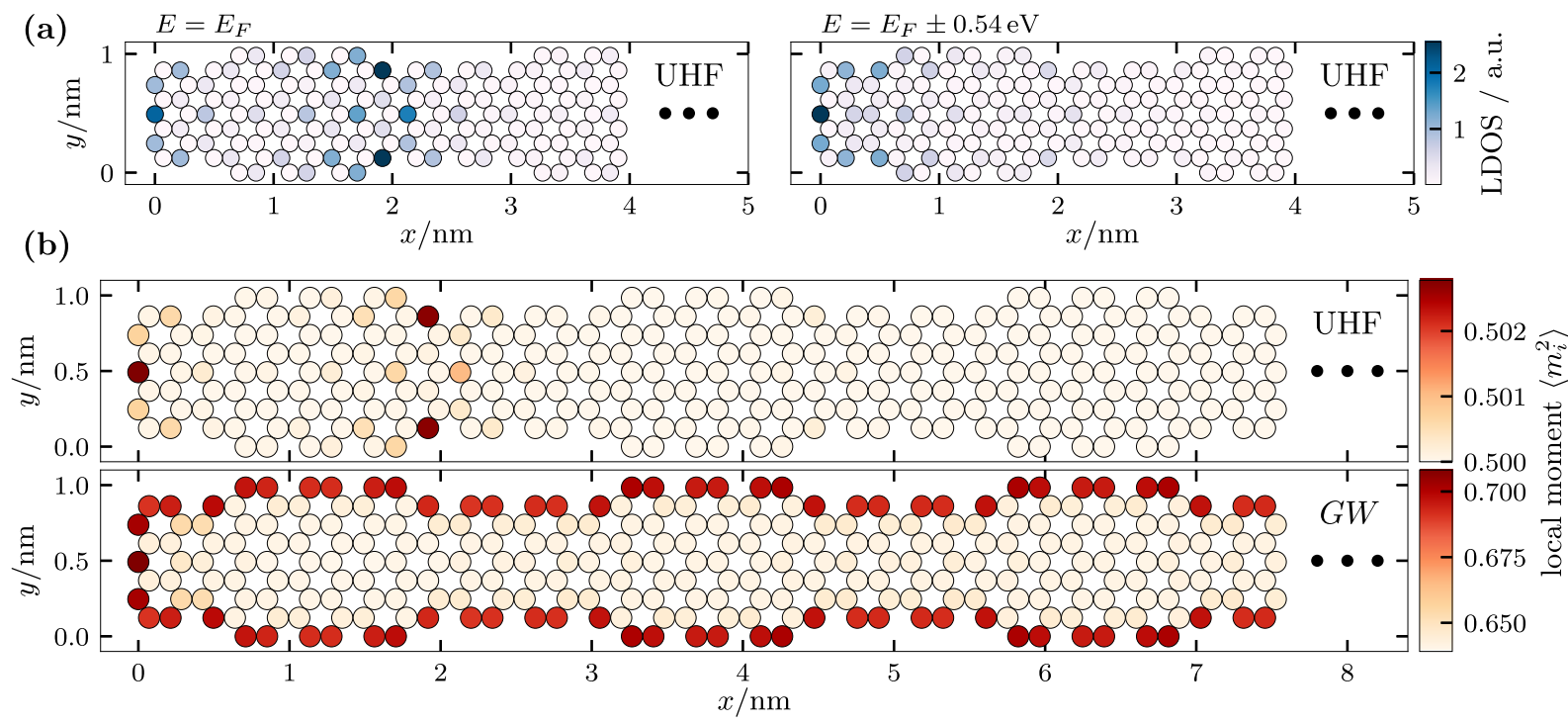

Figure 2. (a) LDOS of the topological end states at $E=E_{\mathrm{F}}$ (left) and $E=E_{\mathrm{F}} \pm 0.54 \mathrm{eV}$ (right) for UHF as shown in Figure 1c. (b) Local moment $\left\langle m_{i}^{2}\right\rangle$, eq 3, of the 7-9-AGNR of Figure 1a calculated within UHF (top) and GW (bottom). Since the ribbon is symmetric, only three of the six unit cells are shown as indicated by the three black dots.

of the atomic sites of the lattice structure, following the procedures described in refs 30 and 31.

Quasiparticle Renormalization. In Figure $1 \mathrm{~b}$ and c, we present differential conductance results for the 7-9-AGNR heterostructure on $\mathrm{Au}(111)$, comparing TB, UHF, and GW simulations to the experiment of ref 18 . Our calculations were performed for a half-filled system containing six unit cells as shown in Figure 1a. In accordance with the measurements, the results for the bulk (end) calculation are averaged over the area marked by the red (blue) cross. Note that, in the experiment, the ribbon was slightly doped, shifting the differential conductance spectrum to higher energies. For comparison, the experimental data were shifted so that the zero-energy peaks of theory and experiment match. In the measurements, a band gap of $E_{\mathrm{g} \text {, bulk }}^{\exp }=0.74 \mathrm{eV}$ between the bulk bands is observed, whereas the gap between the nonzero-energy end peaks is $E_{\mathrm{g} \text {, end }}^{\exp }=1.32 \mathrm{eV}$. The $\mathrm{TB}$ approximation vastly underestimates the gaps, with $E_{\mathrm{g} \text {, bulk }}^{\mathrm{TB}}=0.52 \mathrm{eV}$ and $E_{\mathrm{g} \text {, end }}^{\mathrm{TB}}=$ $1.08 \mathrm{eV}$, respectively. In addition to the two bands in the bulk region, an additional unphysical zero-energy mode appears in the TB solution.

Next, we include mean-field effects within the UHF approximation. This does not lead to a considerable energy renormalization for the bulk and end states but to a splitting of all three topological states that are localized at the end of the heterostructure; compare Figure 1c. The behavior of the two nonzero-energy end peaks is particularly surprising. While the splitting of the zero-energy edge peaks in ZGNRs is wellknown $^{32}$ and is attributed to magnetic instabilities at the Fermi level, ${ }^{33}$ the splitting of states at nonzero energy cannot be understood in this picture. In the experimental data where the system is on top of a screening $\mathrm{Au}(111)$ surface, this effect is not observed. However, metallic substrates are known to suppress the splitting of zero-energy states in finite length pristine AGNRs ${ }^{34}$ compared to insulating substrates. ${ }^{35}$ The UHF result indicates that a splitting of all three end peaks, as seen in Figure 1c, will emerge in measurements for an insulated heterojunction.

Finally, including quasiparticle corrections within the $G W$ approximation results in a considerable correlation-induced renormalization of both the bulk and end states. The observed gaps of $E_{\mathrm{g} \text {, bulk }}^{G W}=0.71 \mathrm{eV}$ and $E_{\mathrm{g}, \text { end }}^{G W}=1.35 \mathrm{eV}$ are in excellent agreement with the experimental findings. Additionally, $G W$ correctly reduces the unphysical zero-energy contribution in the bulk and prevents the splitting of the end states through self-consistent screening. One should note that the extreme broadening of the upper bulk band seen in the experiment is not captured by our simulations. The origin of this broadening is unclear at present; however, it is probably caused by the experimental setup, that is, the substrate or the tip.

Local Correlations. To understand the mechanisms causing the above-mentioned renormalization and splitting of the topological states, we next consider local correlations and magnetic polarizations. In Figure $2 b$, we compare the local moment at site $i$ :

$$
\left\langle\hat{m}_{i}^{2}\right\rangle=\left\langle\left(\hat{n}_{\uparrow, i}-\hat{n}_{\downarrow, i}\right)^{2}\right\rangle=\rho_{i}-2 D_{i}
$$

from UHF- and $G W$-simulations for the same system as in Figure 1. The local moment quantifies the local interaction energy and is a measure of the local magnetic polarization of the system. It is directly related to the local density $\rho_{i}$ and double occupation $D_{i}$ at site $i$ (cf. eq 3 ), which are strongly affected by electronic correlations. Consequently, the local moment is, in general, higher for $G W$ than for UHF. In the latter case, the local moment is peaked exactly at the sites where the zero-energy end state is localized, which can be confirmed by comparing to the LDOS in Figure 2a. Considering the splitting of the zero-energy end peak in Figure 1c, this is in agreement with previous mean-field calculations for ZGNRs ${ }^{33,36}$ where the magnetic instability of the zero-energy edge state gives rise to an antiferromagnetic ordering at opposing zigzag edges. However, such an instability does not occur for the nonzero-energy end states for which the local distribution only partially coincides with the local moment; compare Figure 2a. Instead, the splitting of these states observed in Figure 1c originates from their hybridization with the zero-energy zigzag state that is further investigated in the discussion of Figure 4. 
Strikingly, for $G W$, the local moment is increased on all edges of the heterostructure, which coincides with the regions where the topological states are localized; compare Figure $4 \mathrm{~b}$. Consequently, the renormalization of the topological bulk and end peaks can be attributed to strongly localized correlations at the edges of the heterostructure. The topological states that extend across the boundary of the heterostructure result in increased magnetic polarization even at the armchair edges of the ribbon. This surprising finding is in contrast to the prediction of the UHF simulation in this work and other meanfield theory results ${ }^{29,33,37-40}$ where typically considerable magnetic polarization is only observed at the zigzag edges of GNRs.

Finite Size Effects. The GNR heterostructure discussed in Figures 1 and 2 contains six unit cells, exactly as in the experiments. In the following, we explore the effect of the system size on the topological states. In Figure 3, the local

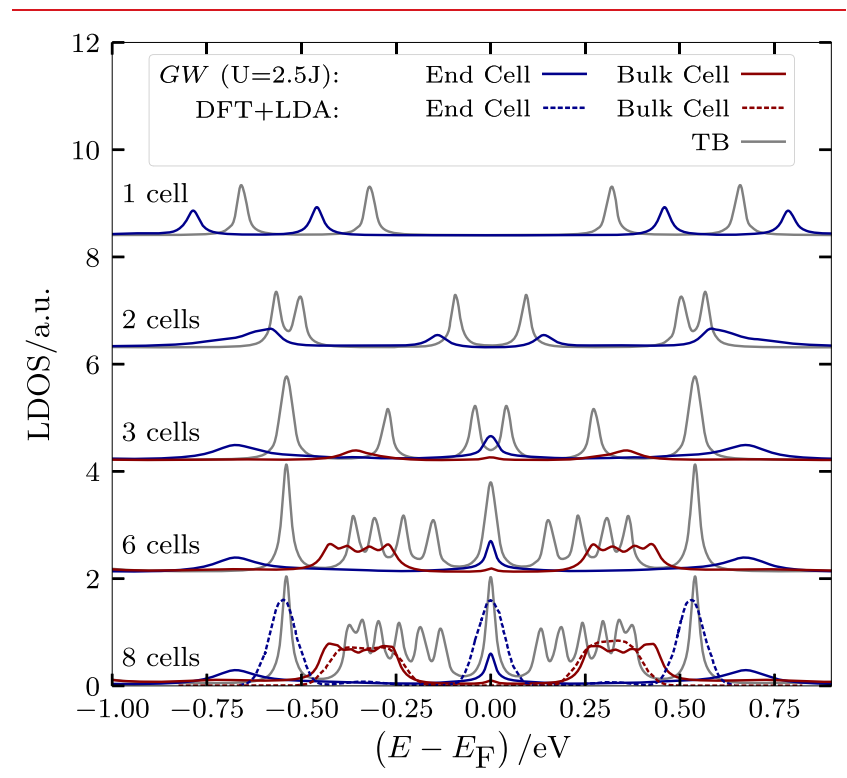

Figure 3. LDOS for 7-9-AGNR heterostructures consisting of one to eight unit cells. The case of six unit cells is the one shown in Figure 1a. Red (blue) lines: bulk (end) cell results as shown in Figure la. Solid (dashed): $G W$ (LDA-DFT) calculations. The DFT results are taken from ref 18 . Solid gray lines: total DOS from TB calculations. The lines for different systems are shifted vertically for better comparison.

density of states (LDOS) of heterostructures containing one to eight unit cells is shown for the bulk and end cells comparing TB and $G W$ results. For the eight unit cell system, additionally, the LDA-DFT result of ref 18 is plotted to allow for a direct comparison to our results. The observed effects differ for the two regions of the system. The spectral weight of the bulk bands increases with system size. In fact, the TB results, which show the total DOS, indicate that the number of peaks in the bulk bands corresponds to the number of bulk unit cells. This is in agreement with the idea that the bulk bands form by hybridization of heterojunction states of adjacent bulk cells. For $G W$, the energies of the bulk states are renormalized and broadened by electronic correlations, as shown in Figure 1.

In the end cell, the three topological states are stable for systems of three or more unit cells. For these large systems, the states on both ends of the ribbon are separated by bulk cells. However, for smaller systems, the states of opposing termini overlap and result in an additional splitting of the end states. In addition to this topological effect, the energies of the end states are also renormalized due to electronic correlations, in general resulting in higher energies of the states. However, interestingly, for the intermediate system of three unit cells, the splitting of the zero-energy state is reduced for the correlated $G W$ result as compared to TB. This is the result of a competition of both aforementioned effects. Within $G W$, the spatial extension of the zero-energy state is strongly reduced, compare Figure $1 \mathrm{~b}$ and the SI, resulting in a significantly smaller overlap and finite-size splitting of the states on both ends of the system.

Comparing the results of $G W$ and LDA-DFT for the eight unit cell system reveals that LDA-DFT captures reasonably well the renormalization of the bulk band energies, whereas it completely fails to describe the shift of the end states. This indicates that a correct characterization of these topological end states is particularly challenging and requires an accurate description of the underlying electronic correlations.

Topological End States. Since the topological states at the end of the heterostructure are found to be particularly sensitive to electron-electron interactions, it is important to examine the origin of these states in detail. The emergence of topological end states at the termini of GNR heterostructures can be explained by the $\mathbb{Z}_{2}$ invariant and the bulk-boundary correspondence of topological insulator theory. ${ }^{10}$ However, to get a better understanding of their properties, in the following, the specific mechanism that leads to the existence of these multiple end states will be analyzed. For this, in Figure 4, the total DOS (a) and the $\mathrm{d} I / \mathrm{d} V$ maps (b) of the heterostructure containing six unit cells (green) are compared to the same
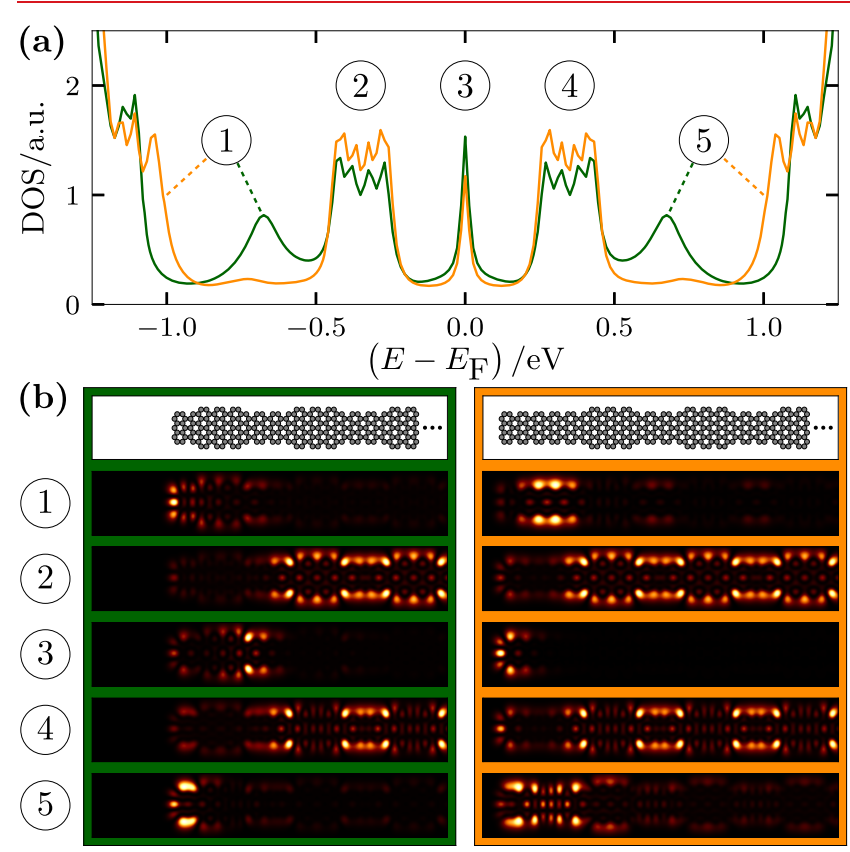

Figure 4. (a) Total DOS calculated with $G W$ for the two systems indicated in the top panel of (b). The green (orange) line corresponds to the left (right) system. The left system contains exactly six unit cells, while the right system is extended additionally by ten zigzag lines on both sides. (b) $d I / d V$ maps for the same two systems. The maps labeled $1-5$ correspond to the labeled peaks in (a). Only a small section of the ribbon is shown indicated by the three black dots in the top panel. 
system with an additional ten zigzag lines on each side of the ribbon (orange) for GW. The two systems are depicted in the topmost panel of Figure $4 \mathrm{~b}$. By comparing the DOS, it stands out that the high-energy end peaks, that are present in the six unit cell system, are strongly suppressed in the longer system. Instead, a new high-energy peak emerges around $\pm 1 \mathrm{eV}$. Additionally, the spectral weight of the bulk bands is increased for the longer system. All these observations can be understood from the $\mathrm{d} I / \mathrm{d} V$ maps of the states in Figure $4 \mathrm{~b}$. For the longer system, the zigzag edge at the end of the system is separated from the heterojunction of the first unit cell by a long 7-AGNR section. Because of this clear separation, the three end states $(1,3,5)$ resemble states observed in pristine 7-AGNR; see SI. Furthermore, the heterojunction states of all six unit cells hybridize to form the bulk bands $(2,4)$. In contrast, for the shorter system, the adjacent states localized at the zigzag edge and at the heterojunctions of the first unit cell can overlap leading to three hybridized states in the termini of the system. As a consequence, the bulk bands are localized only in the four inner bulk unit cells and do not occupy the end cells resulting in a reduced spectral weight in the DOS.

Splitting of peaks induced by the spatial overlap of the associated states typically appears in small finite systems; compare Figure 3 for one unit cell. Strikingly, due to the close proximity of finite substructures, that is, heterojunctions and ribbon edges, the same effect emerges at the termini of large heterostructures containing hundreds to thousands of atoms. Consequently, this observation is not restricted to the present 7-9-AGNR heterostructures. Instead, we predict that similar strongly correlated states exist also in an entire class of systems, which meet the criteria for hosting hybridized end states, that is, possess topological states close to localized edge states. The existence of such states is determined by the topology of the system. $^{10,41,42}$

Summary and discussion. We analyzed the influence of electronic correlations on the topological states of 7-9-AGNR heterostructures on $\mathrm{Au}(111)$. While the general topological structure of the system, previously predicted on the TB level, ${ }^{10}$ remains stable, additional new effects connected to the topological states emerge. Our $G W$ simulations reveal that strong local electronic correlations are present in both the edges of the bulk and the end region of the heterostructure resulting in increased magnetic moments in the zigzag and armchair edges. Strikingly, the spatially confined topological states of the termini are more severely affected by these correlations than the extended topological bulk bands. For the latter, we found, by comparison to our $G W$ results, that LDADFT is able to reproduce the experimental $\mathrm{d} I / \mathrm{d} V$ measurements since quasiparticle corrections are weak in this case. In contrast, the topological end states, emerging due to the hybridization of zigzag-edge and heterojunction states, are strongly renormalized and screened due to electronic correlations, which give rise to a large discrepancy between LDA-DFT and experimental energies. For free-standing heterostructures, we find a new mechanism that leads to the splitting of nonzero-energy states due to hybridization with a zero-energy state. These findings are not restricted to the specific system considered here but instead are expected to be present in similar GNR heterostructures that exhibit strongly localized topological states.

\section{ASSOCIATED CONTENT}

\section{Supporting Information}

The Supporting Information is available free of charge at https://pubs.acs.org/doi/10.1021/acs.nanolett.9b04075.

Details on Green function approach, fitting of Hubbard interaction $U$, screening of zero-energy state, comparison to 7-AGNR states (PDF)

\section{AUTHOR INFORMATION}

\section{Corresponding Author}

*E-mail: bonitz@theo-physik.uni-kiel.de. Phone: +49 (0)431 880-4122. Fax: +49 (0)431 880-4094.

ORCID

Michael Bonitz: 0000-0001-7911-0656

Notes

The authors declare no competing financial interest.

\section{ACKNOWLEDGMENTS}

We acknowledge helpful discussions with Niclas Schlünzen. A.P.J. is supported by the Danish National Research Foundation, Project DNRF103.

\section{REFERENCES}

(1) Stormer, H. L.; Tsui, D. C.; Gossard, A. C. The fractional quantum Hall effect. Rev. Mod. Phys. 1999, 71, 298-305.

(2) Das Sarma, S.; Adam, S.; Hwang, E. H.; Rossi, E. Electronic transport in two dimensional graphene. Rev. Mod. Phys. 2011, 83, 407-470.

(3) Neilson, D.; Perali, A.; Zarenia, M. Manybody electron correlations in graphene. J. Phys.: Conf. Ser. 2016, 702, 012008.

(4) Balzer, K.; Rasmussen, M. R.; Schlünzen, N.; Joost, J.-P.; Bonitz, M. Doublon Formation by Ions Impacting a Strongly Correlated Finite Lattice System. Phys. Rev. Lett. 2018, 121, 267602.

(5) Cao, Y.; Fatemi, V.; Demir, A.; Fang, S.; Tomarken, S. L.; Luo, J. Y.; Sanchez-Yamagishi, J. D.; Watanabe, K.; Taniguchi, T.; Kaxiras, E.; Ashoori, R. C.; Jarillo-Herrero, P. Correlated insulator behaviour at half-filling in magic-angle graphene superlattices. Nature 2018, 556, $80-84$.

(6) Cao, Y.; Fatemi, V.; Fang, S.; Watanabe, K.; Taniguchi, T.; Kaxiras, E.; Jarillo-Herrero, P. Unconventional super-conductivity in magic-angle graphene super-lattices. Nature 2018, 556, 43-50.

(7) Kerelsky, A.; McGilly, L. J.; Kennes, D. M.; Xian, L.; Yankowitz, M.; Chen, S.; Watanabe, K.; Taniguchi, T.; Hone, J.; Dean, C.; Rubio, A.; Pasupathy, A. N. Maximized electron interactions at the magic angle in twisted bilayer graphene. Nature 2019, 572, 95-100.

(8) Cai, J.; Pignedoli, C. A.; Talirz, L.; Ruffieux, P.; Söde, H.; Liang, L.; Meunier, V.; Berger, R.; Li, R.; Feng, X.; Müllen, K.; Fasel, R. Graphene nano-ribbon heterojunctions. Nat. Nanotechnol. 2014, 9, 896-900.

(9) Chen, Y.-C.; Cao, T.; Chen, C.; Pedramrazi, Z.; Haberer, D.; de Oteyza, D. G.; Fischer, F. R.; Louie, S. G.; Crommie, M. F. Molecular bandgap engineering of bottom-up synthesized graphene nanoribbon heterojunctions. Nat. Nanotechnol. 2015, 10, 156-160.

(10) Cao, T.; Zhao, F.; Louie, S. G. Topological Phases in Graphene Nanoribbons: Junction States, Spin Centers, and Quantum Spin Chains. Phys. Rev. Lett. 2017, 119, 076401.

(11) Jacobse, P. H.; Kimouche, A.; Gebraad, T.; Ervasti, M. M.; Thijssen, J. M.; Liljeroth, P.; Swart, I. Electronic components embedded in a single graphene nanoribbon. Nat. Commun. 2017, 8, 119.

(12) Ma, C.; Liang, L.; Xiao, Z.; Puretzky, A. A.; Hong, K.; Lu, W.; Meunier, V.; Bernholc, J.; Li, A.-P. Seamless Staircase Electrical Contact to Semiconducting Graphene Nanoribbons. Nano Lett. 2017, 17, 6241-6247. 
(13) Wang, S.; Kharche, N.; Costa Girão, E.; Feng, X.; Müllen, K.; Meunier, V.; Fasel, R.; Ruffieux, P. Quantum Dots in Graphene Nanoribbons. Nano Lett. 2017, 17, 4277-4283.

(14) Rizzo, D. J.; et al. Length-Dependent Evolution of Type II Heterojunctions in Bottom-Up-Synthesized Graphene Nanoribbons. Nano Lett. 2019, 19, 3221-3228.

(15) Hasan, M. Z.; Kane, C. L. Colloquium: Topological insulators. Rev. Mod. Phys. 2010, 82, 3045-3067.

(16) Asbóth, J. K.; Oroszlány, L.; Pályi, A. A Short Course on Topological Insulators: Band Structure and Edge States in One and Two Dimensions; Lecture Notes in Physics; Springer: Cham, 2016; Vol. 919.

(17) Gröning, O.; Wang, S.; Yao, X.; Pignedoli, C. A.; Borin Barin, G.; Daniels, C.; Cupo, A.; Meunier, V.; Feng, X.; Narita, A.; Müllen, K.; Ruffieux, P.; Fasel, R. Engineering of robust topological quantum phases in graphene nanoribbons. Nature 2018, 560, 209-213.

(18) Rizzo, D. J.; Veber, G.; Cao, T.; Bronner, C.; Chen, T.; Zhao, F.; Rodriguez, H.; Louie, S. G.; Crommie, M. F.; Fischer, F. R. Topological band engineering of graphene nanoribbons. Nature 2018, $560,204$.

(19) Lado, J. L.; Ortiz, R.; Fernández Rossier, J. Emergent quantum matter in graphene nanoribbons. arXiv:1909.00258 [cond-mat] 2019.

(20) Stefanucci, G.; van Leeuwen, R. Nonequilibrium Many-Body Theory of Quantum Systems: A Modern Introduction; Cambridge University Press: Cambridge, 2013.

(21) Schlünzen, N.; Hermanns, S.; Scharnke, M.; Bonitz, M. Ultrafast dynamics of strongly correlated fermions - Nonequilibrium green functions and selfenergy approximations. J. Phys.: Condens. Matter 2019. DOI: $10.1088 / 1361-648 X / a b 2 d 32$

(22) Haug, H.; Jauho, A.-P. Quantum Kinetics in Transport and Optics of Semiconductors; Springer: Berlin, Heidelberg, 2008.

(23) Balzer, K.; Bonitz, M. Nonequilibrium Green's Functions Approach to Inhomogeneous Systems; Springer: Berlin, Heidelberg, 2013.

(24) Reich, S.; Maultzsch, J.; Thomsen, C.; Ordejón, P. Tightbinding description of graphene. Phys. Rev. B 2002, 66, 035412.

(25) Xu, Z.; Zheng, Q.-S.; Chen, G. Elementary building blocks of graphene-nanoribbon-based electronic devices. Appl. Phys. Lett. 2007, $90,223115$.

(26) Sevinçli, H.; Topsakal, M.; Ciraci, S. Superlattice structures of graphene-based armchair nanoribbons. Phys. Rev. B 2008, 78, 245402.

(27) Tao, C.; Jiao, L.; Yazyev, O. V.; Chen, Y.- C.; Feng, J.; Zhang, X.; Capaz, R. B.; Tour, J. M.; Zettl, A.; Louie, S. G.; Dai, H.; Crommie, M. F. Spatially resolving edge states of chiral graphene nanoribbons. Nat. Phys. 2011, 7, 616-620.

(28) Joost, J.-P.; Schlünzen, N.; Bonitz, M. Femtosecond Electron Dynamics in Graphene Nanoribbons - A Nonequilibrium Green Functions Approach Within an Extended Hubbard Model. Phys. Status Solidi B 2019, 256, 1800498.

(29) Yazyev, O. V.; Capaz, R. B.; Louie, S. G. Theory of magnetic edge states in chiral graphene nanoribbons. Phys. Rev. B 2011, 84, 115406.

(30) Tersoff, J.; Hamann, D. R. Theory of the scanning tunneling microscope. Phys. Rev. B 1985, 31, 805-813.

(31) Meunier, V.; Lambin, P. Tight-Binding Computation of the STM Image of Carbon Nanotubes. Phys. Rev. Lett. 1998, 81, 55885591.

(32) Magda, G. Z.; Jin, X.; Hagymási, I.; Vancsó, P.; Osváth, Z.; Nemes-Incze, P.; Hwang, C.; Biró, L. P.; Tapasztó, L. Roomtemperature magnetic order on zigzag edges of narrow graphene nano-ribbons. Nature 2014, 514, 608-611.

(33) Pisani, L.; Chan, J. A.; Montanari, B.; Harrison, N. M. Electronic structure and magnetic properties of graphitic ribbons. Phys. Rev. B 2007, 75, 064418.

(34) van der Lit, J.; Boneschanscher, M. P.; Vanmaekelbergh, D.; Ijäs, M.; Uppstu, A.; Ervasti, M.; Harju, A.; Liljeroth, P.; Swart, I. Suppression of electron-vibron coupling in graphene nanoribbons contacted via a single atom. Nat. Commun. 2013, 4, 2023.
(35) Wang, S.; Talirz, L.; Pignedoli, C. A.; Feng, X.; Müllen, K.; Fasel, R.; Ruffieux, P. Giant edge state splitting at atomically precise graphene zigzag edges. Nat. Commun. 2016, 7, 11507.

(36) Feldner, H.; Meng, Z. Y.; Lang, T. C.; Assaad, F. F.; Wessel, S.; Honecker, A. Dynamical Signatures of Edge-State Magnetism on Graphene Nanoribbons. Phys. Rev. Lett. 2011, 106, 226401.

(37) Fernández-Rossier, J.; Palacios, J. J. Magnetism in Graphene Nanoislands. Phys. Rev. Lett. 2007, 99, 177204.

(38) Feldner, H.; Meng, Z. Y.; Honecker, A.; Cabra, D.; Wessel, S.; Assaad, F. F. Magnetism of finite graphene samples: Mean-field theory compared with exact diagonalization and quantum Monte Carlo simulations. Phys. Rev. B 2010, 81, 115416.

(39) Golor, M.; Koop, C.; Lang, T. C.; Wessel, S.; Schmidt, M. J. Magnetic Correlations in Short and Narrow Graphene Armchair Nanoribbons. Phys. Rev. Lett. 2013, 111, 085504.

(40) Ortiz, R.; García-Martínez, N. A.; Lado, J. L.; FernándezRossier, J. Electrical spin manipulation in graphene nanostructures. Phys. Rev. B 2018, 97, 195425.

(41) Lee, Y.-L.; Zhao, F.; Cao, T.; Ihm, J.; Louie, S. G. Topological Phases in Cove-Edged and Chevron Graphene Nanoribbons: Geometric Structures, Z2 Invariants, and Junction States. Nano Lett. 2018, 18, 7247-7253.

(42) Lin, K.-S.; Chou, M.-Y. Topological Properties of Gapped Graphene Nanoribbons with Spatial Symmetries. Nano Lett. 2018, 18, 7254-7260. 